\title{
A BLUNTED CONE IN A SUPERSONIC HIGH-ENTHALPY NONEQUILIBRIUM AIR FLOW
}

\author{
V.I. Sakharov ${ }^{1}$, V. V. Shtapov ${ }^{2}$, E. B. Vasilevskiy ${ }^{2}$, \\ and B. E. Zhestkov ${ }^{2}$ \\ ${ }^{1}$ Institute of Mechanics, M. V. Lomonosov Moscow State University \\ 1 Michurinskii Prosp., Moscow 119192, Russia \\ ${ }^{2}$ Central Aerohydrodynamic Institute (TsAGI) \\ 1 Zhukovsky Str., Zhukovsky, Moscow Region 140180, Russia
}

\begin{abstract}
A calculation and experimental study was conducted with the flow, heat flux, and pressure distributions over the front and side surfaces of a blunt cone in a nonequilibrium high-enthalpy $\left(h_{0}=25 \mathrm{MJ} / \mathrm{kg}\right)$ supersonic $(\mathrm{M}=4)$ air flow. The experiments were performed in a VAT-104 wind tunnel (WT), TsAGI. The nose part of the model with a small-radius nose $R_{w}=10 \mathrm{~mm}$ and half angle $\theta=10^{\circ}$ was inside the "Mach cone" of the underexpanded jet flowing out from the WT nozzle. Numerical and experimental results are in good agreement.
\end{abstract}

\section{INTRODUCTION}

Simulation of thermochemical interaction between high-enthalpy flows of air and other gases and heat-protective materials (HPM) in high-enthalpy WTs for flight conditions of bodies with hypersonic speeds in atmosphere of the Earth, Mars, and other planets is among key problems of air thermodynamics [1].

Inductive (electrodeless) plasmatorches which produce high-enthalpy chemically pure gas flows have a good potentiality in such simulation, particularly, in respect to the thermal effect of heterogeneous catalytic reactions of atomic recombination [2-10]. Such WTs are utilized, in particular, to carry out ground experimental researches of the thermal protection for the flight conditions of hypersonic aircraft (HA) in the upper regions of the Earth atmosphere (about $60-100 \mathrm{~km}$ ). These WTs enable to study HPM properties such as surface catalyticity, emissivity, and thermal stability in flows of various gases. Such works have been performed for many years with inductive plasmatorches in TsAGI (VAT-104 WT), IPMech RAS (IPG-4), TsNIIMASh, Von-Karman Institute, etc.

This is an Open Access article distributed under the terms of the Creative Commons Attribution License 4.0, which permits unrestricted use, distribution, and reproduction in any medium, provided the original work is properly cited. 
The IPG-4 plasmatorch (IPMech RAS) of $100 \mathrm{~kW}$ permits having both subsonic and supersonic high-enthalpy flows of air and other gases within a wide range of stagnation temperature and pressure [4-8], whereas the VAT-104 WT (TsAGI) is purposed to produce supersonic $(\mathrm{M}=4-8)$ high-enthalpy flows. Note that the simulation of heat exchange in the inductive plasmatorch at the stagnation point for the conditions of hypersonic flights suggests, among other factors, the equality of total enthalpies and pressures on the surface of bodies in the ground experiment and in the hypersonic flight $[2,3,9,10]$. Thus, supersonic nozzles in the WT enable to expand the area of parameters for heat exchange simulation in the ground experiment.

To analyze the experimental data, in particular, the catalytic properties of modern HTMs, and to transfer them in full-scale conditions of the hypersonic flight of bodies in the atmosphere, it is necessary to know in detail the flows realized in the experiment, as well as the physical processes taking place in the WT and on model surfaces. To solve these and other challenges of modern aerodynamic experiments, one should simulate numerically the supersonic flows of chemically reacting gases and heat exchange for specific experimental conditions, including those implemented in the VAT-104 WT (TsAGI) [10]. Today, there is a serious gap between high-level mathematical and physical models applied in hypersonic aerodynamics and approximate models utilized for the analysis of WT flows. Numerical simulation for subsonic and supersonic regimes was successfully fulfilled.

In a number of cases, flows in WTs are more complicated in respect to the analysis of obtained experimental data than the hypersonic flows near bodies incoming into the atmosphere. The WT gas heated in the heater up to high temperature cools down when the flow speed rises as the flow goes out through the nozzle into the working chamber. The gas temperature rises again behind the shock wave induced by the body. At the same time, in the flight, the gas reaches high values only after passing the shock wave induced by the body. Consequently, the gas properties near the model in the WT and near the HA surface in the flight may differ significantly at the same values of stagnation enthalpy and pressure on the body surface. In recent years, the flows in the discharge channel of the IPG-4 plasmatorch and the VAT-104 WT heater were studied in the context of Navier-Stokes equations and simplified Maxwell equations, involving chemically and thermally nonequilibrium models of the gas medium, as well as the flows in flowing-out underexpanded jets of air plasma. The study was carried out within a wide range of supersonic modes of WTs: air flow rates, values of the power contributed in plasma, reverse pressures in decompression chambers, and sizes of outlet cross sections and sonic and supersonic nozzles. The technology of calculation of such flows is based on a software system of numerical integration of Navier-Stokes equations and special programs-generators which include data bases for thermodynamic and transfer properties of individual gaseous substances (HIGHTEMP) created in the Institute of Mechanics MSU [8]. 
In the present paper, numerical investigation was done for the processes and flows in a chemically nonequilibrium model of the gas medium, in the heater and channel of VAT-104 WT (TsAGI) for the air dynamics of flowing-out underexpanded high-enthalpy air jets and for the model tested in the experiments; the results are compared with the experiment.

The aim of this work was to determine the heat flux and pressure distribution over the front and side surfaces of the blunt cone in the nonequilibrium high-enthalpy $\left(h_{0}=25 \mathrm{MJ} / \mathrm{kg}\right)$ supersonic $(\mathrm{M}=4)$ air flow. Experimental investigations were carried out in the VAT-104 TsAGI. The front part of the model with a small-radius nose $R_{w}=10 \mathrm{~mm}$ and half angle $\theta=10^{\circ}$ was inside the "Mach cone" of the underexpanded jet flowing out from the WT nozzle. It is known that the disturbances occurring in the working chamber of the hypersonic WT cannot penetrate into the area inside the "Mach cone" (toward the nose part of the model): this fact facilitates interpretation of the test results in comparison with the experiments in subsonic WTs.

In order to measure the heat flux distribution, heat-capacity heat-flux probes were utilized (copper inserts thermally insulated from the model body). These probes were calibrated by a standard technique on a special calibrating heat-flux facility ETGU (TsAGI).

To measure the pressure distribution, pressure gages were used; they were located directly inside the model. It allowed significant reducing of the pressure measurement duration in the flow by means of utilization of short routes from the receiving orifice to the pressure gage. Short duration of the measurement (test) provided complete and repetitive safety of the model under the conditions of a strong action of the heat flux on it.

A special high-speed mechanism of model introduction into the high-enthalpy flow was developed for the short-run test in the WT (the time of jet crossing was below $0.2 \mathrm{~s}$ ).

\section{EXPERIMENTAL EQUIPMENT}

\subsection{VAT-104 Wind Tunnel}

The basic elements of the WT VAT-104 are the gas heater, nozzle, working chamber, and a system of pumping-out with a vacuum capacity (Fig. 1). Figure 2 shows the basic schematic of the VAT-104 WT.

The gas is heated with a high-frequency (HF) inductive heater, which permits to have a spectrally pure high-enthalpy gas flow. The heaters of this type feature high stability and repetitive accuracy of enthalpy, total stagnation pressure, and heat flux from one test to another (the parameter deviations from assigned values do not exceed $3 \%$ ). 


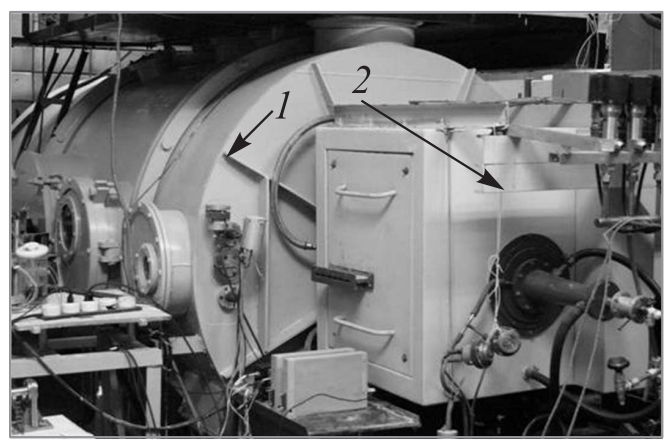

Figure 1 A photograph of VAT-104 WT: 1 - working chamber; and $2-\mathrm{HF}$ inductive heater

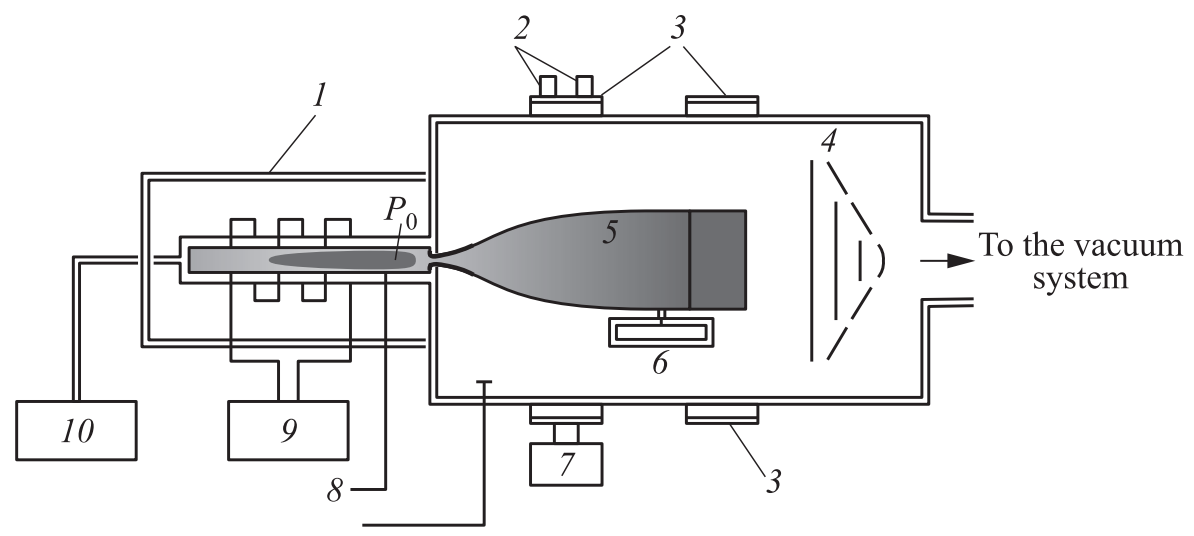

Figure 2 The basic schematic of VAT-104 WT: 1 - induction heater; 2 - CCD (charge-coupled device) camera; 3 - optical windows; 4 - cooler; 5 - model; 6 input mechanism; 7 - AGA thermovision; 8 - pressure gauges; $9-\mathrm{HF}$ generator; and 10 - gas supply

The inductor is fed from the HF generator VChI-4-160/1.76. The generator is of a two-loop configuration, with controlled feedback. The generator power in the anode circuit is up to $W_{a}=240 \mathrm{~kW}$, the oscillating power is up to $W_{\sim}=160 \mathrm{~kW}$, and the frequency is $\nu=1.76 \mathrm{MHz}$. Standard discharge ignition is carried out in an argon medium as the discharge chamber is vacuumed to the pressure $P_{0}=5-30 \mathrm{~Pa}$. Then the working gas is supplied, its flow rate and heater power are increased smoothly. The flow rate of the working gas is controlled by a throttle system, manually or automatically (a program sets the mode). The mode onset time is from 10 to $30 \mathrm{~s}$. Various gases such as nitrogen, air, argon, 
argon-oxygen mixture, etc. can be used as the working substance. This time, air was used.

The plasmatorch heats the working gas up to the temperature of $T_{0}=5,000$ $8,000{ }^{\circ} \mathrm{C}$ (the stagnation enthalpy is $i_{0}=10-40 \mathrm{MJ} / \mathrm{kg}$ ) as its flow rate is up to $G=4 \mathrm{~g} / \mathrm{s}$ and the total pressure is up to $P_{0}=50 \mathrm{kPa}(0.5 \mathrm{bar})$. The main parameters of the heater operation in the VAT-104 WT are studied by contact and optical diagnostic techniques. It is found that the vibration and rotational temperatures in the settling chamber coincide within the experimental error limits (10\%).

The results of heat flux measurements made with calorimeters and sensors with coatings of extremely high and extremely low catalytic activity enabled to establish that as the anode voltage was $U=8.5-10.5 \mathrm{kV}$ and the pressure in the heater was $P_{0}=(10-40) \mathrm{kPa}$; the flow enthalpy was $i_{0}=10-40 \mathrm{MJ} / \mathrm{kg}$; air dissociation degree was $\alpha=0.5-0.9$; and the power transferred to the working gas flow from the heater was $W_{f} \approx 50 \mathrm{~kW}$.

With the lower heater power, lower flow parameters can be realized: the pressure of $P_{0}=3-6 \mathrm{kPa}, i_{0}=10-20 \mathrm{MJ} / \mathrm{kg}$, and $\alpha=0.1-0.3$.

The working cell is an Eiffel chamber. It is a cylinder with a diameter of $1.6 \mathrm{~m}$ and a length of $4 \mathrm{~m}$. The inductive heater with a water-cooled nozzle is connected to the front-end face of the working chamber. The back-end face is connected to the vacuum capacity via a vacuum lock.

Inside the working cell, there is a fast-acting mechanism of model introduction into the flow and a heat exchanger which cools down the high-temperature gas jet after the model is immersed in flow. The model was introduced into the flow when the assigned stationary flow mode was reached in the WT (Fig. 3).
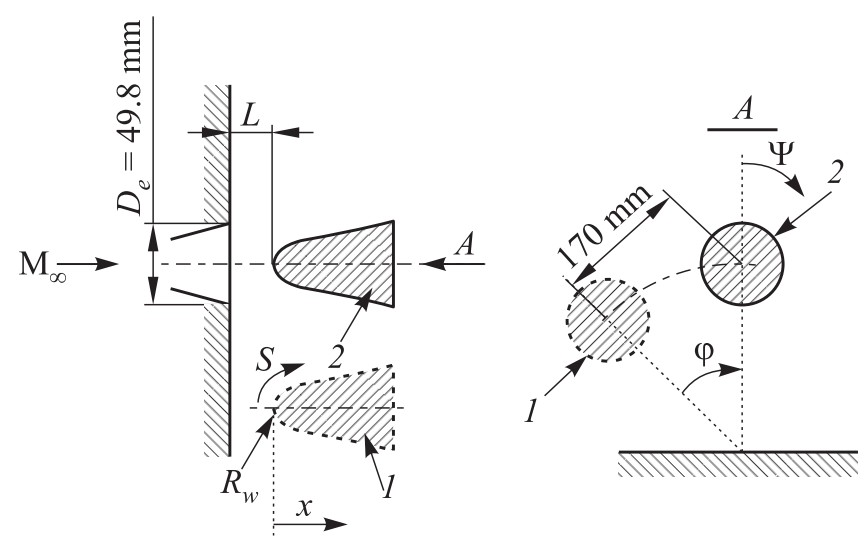

Figure 3 Fast-acting input mechanism before (1) and after (2) model introduction into the flow 
In this research, the Laval nozzle was utilized; it had the throat radius $R^{*}$ $=7.5 \mathrm{~mm}$, the outlet section radius $R_{e}=24.9 \mathrm{~mm}$, and the half angle in the supersonic part of $15^{\circ}$. The pressure in the working part of VAT-104 may vary from 1 to 1,000 $\mathrm{Pa}$, depending on the quantity and type of involved pumps and gas flow rates. In these experiments, the jet flowing out from the nozzle of VAT104 is underexpanded. Mach numbers $M=4-8$ can be reached in the jet at various distances from the nozzle exit.

The maximum duration of the test in VAT-104 is limited by the possibility of working chamber cooling; it varies from $20 \mathrm{~min}$ as the pressure in the settling chamber is $P_{0} \approx 50 \mathrm{kPa}$ to $2 \mathrm{~h}$ at $P_{0} \approx 5 \mathrm{kPa}$. The heat flux to the cold wall $\left(T_{w}=18{ }^{\circ} \mathrm{C}\right)$ at the stagnation point of the flat-end face of the streamwisealigned cylinder with a radius of $R=35 \mathrm{~mm}$ is $q_{0}=100-1500 \mathrm{~kW} / \mathrm{m}^{2}$, and the radiation-equilibrium temperature of the surface may reach $T_{r}=1750{ }^{\circ} \mathrm{C}$.

Normally, deviations of the enthalpy from one test to another do not exceed $3 \%$. In repetitive tests, the error of heat flux determination is $5 \%-10 \%$.

\section{$2.2 \quad$ Model}

The methodical model purposed for the distribution of the heat flux density is made of stainless steel and has a shape of a blunt cone which is identical to the standard sample shape. The photo of the model is given in Fig. 4.

There are nine calorimetric heat-flux gages on the model; they are equipped with thermoanisotropic bushings and a thermocouple to measure the temperature of their cold junction. The locations of the heat flux probes are shown in Figs. 4 and 5.

The heat flux probe has a brass calorimeter shaped as a small cylinder, its diameter is $1.5 \mathrm{~mm}$ and its length is $4 \mathrm{~mm}$. The thermoelectrode consists of

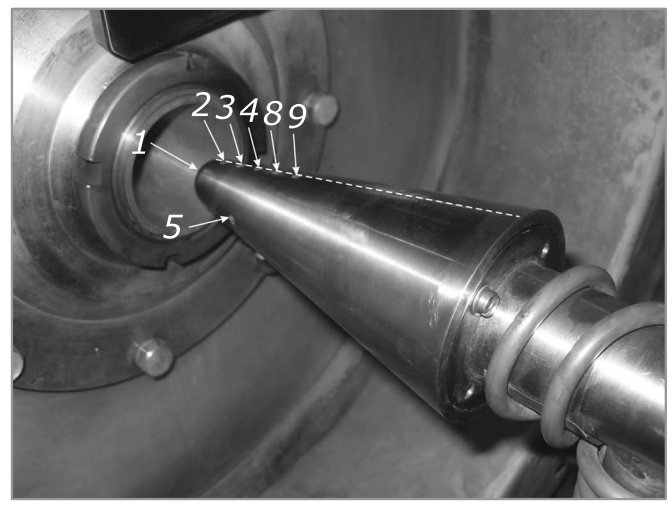

Figure 4 Model photograph 


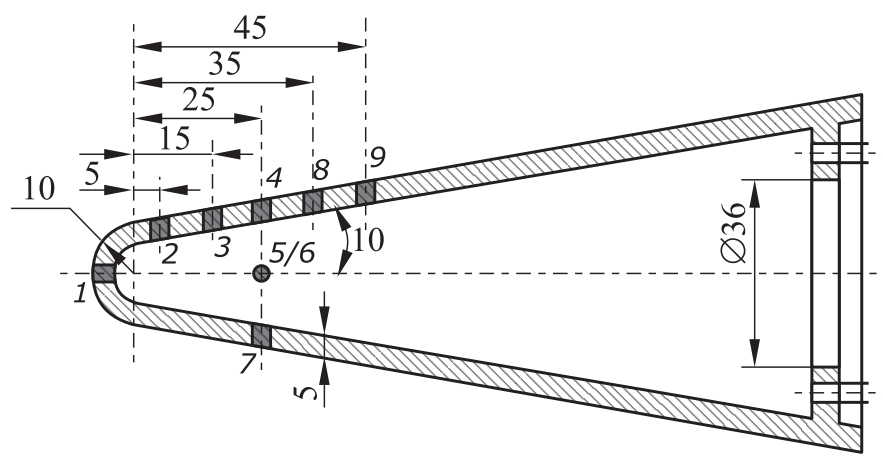

Figure 5 Locations of the heat flux probes 1-9 (see Table 1). Dimensions are in millimeters

Table 1 Locations of the heat flux probes

\begin{tabular}{cccr}
\hline Probe number & $x, \mathrm{~mm}$ & $S, \mathrm{~mm}$ & $\psi$ \\
\hline 1 & 0 & 0.0 & $0^{\circ}$ \\
2 & 15 & 20.8 & $0^{\circ}$ \\
3 & 25 & 30.9 & $0^{\circ}$ \\
4 & 35 & 41.1 & $0^{\circ}$ \\
5 & 35 & 41.1 & $270^{\circ}$ \\
6 & 35 & 41.1 & $90^{\circ}$ \\
7 & 35 & 41.1 & $180^{\circ}$ \\
8 & 45 & 51.2 & $0^{\circ}$ \\
9 & 55 & 61.4 & $0^{\circ}$ \\
\hline
\end{tabular}

"chromel" and "copel" wires, $0.1 \mathrm{~mm}$ in diameter, which are welded to the back side of the cylinder (dot welding). All thermocouple cables are connected to the connector which is located in the aft body of the model.

A heat-insulating bushing, which presents a tight spiral of brass foil and a thin glass-cloth band, serves for thermal insulation of the calorimeter from the model walls. Spiral turns are glued with a high-temperature glue VK-21 (heat resistance up to $700-800^{\circ} \mathrm{C}$ ). Owing to such a probe design, the temperature distribution by the cylinder depth and long turns of the brass foil are approximately similar (especially, on the spiral turns adjoining the calorimeter) (Fig. 6).

At the same time, the effective thermal conductivity across the spiral turns is not high because of the low thermal conductivity of the glass cloth and glue. It permits to linearize probe's characteristics and, hence, increase the potential measurement time up to $3-4 \mathrm{~s}$. In order to sufficiently reduce leakages resulting from the plasma flow, the probes were coated with an insulator (thin, approximately $2 \mu \mathrm{m}$, layer of gel $\mathrm{SiO}_{2}$ ). It enabled to illuminate discharge current to 

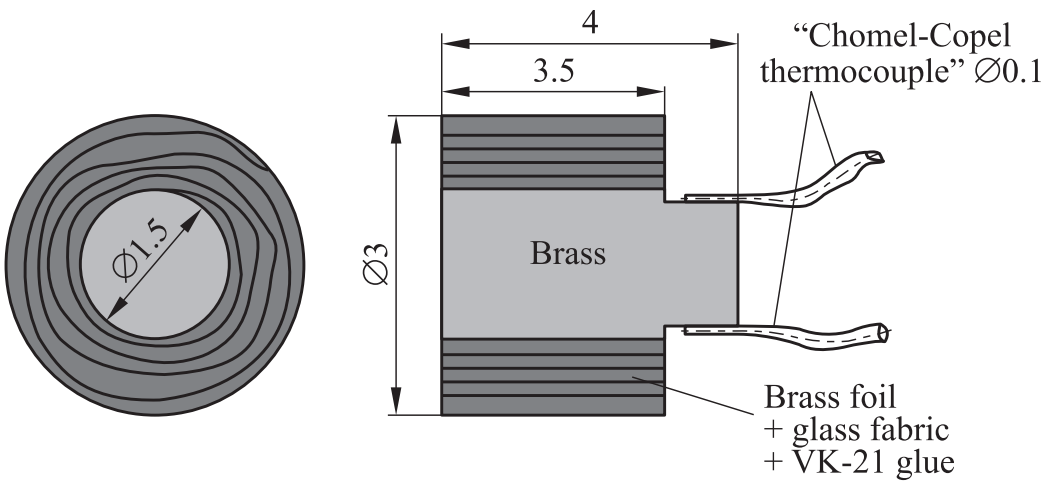

Figure 6 Heat flux probe design. Dimensions are in millimeters

the gages and to obtain more "pure" signals, whereas the influence of the $\mathrm{SiO}_{2}$ layer on $K_{1}$ was insignificant.

Each heat-flux probe was graduated many times in the ITGU pulse thermal calibrating facility based at NIO-8 TsAGI. The facility permits to supply stepfunction signals $q_{0}$ of the convective heat flux with known magnitudes. The probe calibrating factor $K_{1}$ is determined by the response signal $U_{0}(\tau)$ :

$$
K_{1}=\frac{q}{d U / d \tau}
$$

Later, these calibrating factors will be utilized to determine the heat flux density during the tests of the model in the VAT-104 in the ITGU pulse thermal calibrating facility based at NIO-8 TsAGI:

$$
q=k \frac{d U}{d \tau}
$$

where $k=K_{1} K_{2}$. Here, $U$ is the probe signal in millivolts recorded during the tests and $K_{2}$ is the factor of the amplifier of the signals established in the VAT-104 WT.

The model for pressure distribution measurement was equipped with five small-scale inductive gages (type DMI-0.6-III) for the rated range of $0.6 \mathrm{kgs} / \mathrm{cm}^{2}$. The probes were preliminary mounted on a special unit installed inside the model body. Figure 7 presents the photo of the model with the probes inside.

The receiving orifices of the gages $\left(d_{\text {in }}=1 \mathrm{~mm}\right)$ lied on one line along the model generatrix. The coordinates of the pressure sampling points are listed in Table 2.

The pedestal areas of the gages are connected with a sealed channel; this channel is a carrying collector for every gage, it has a special outlet tube, which 


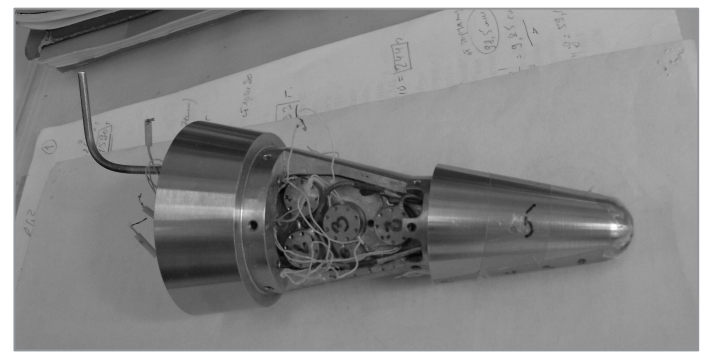

Figure 7 Model for pressure distribution measurement

Table 2 Orifices locations

\begin{tabular}{ccc}
\hline Probe number & $x, \mathrm{~mm}$ & $S, \mathrm{~mm}$ \\
\hline 1 & 0 & 0.0 \\
2 & 15 & 20.8 \\
3 & 25 & 30.9 \\
4 & 35 & 41.1 \\
5 & 55 & 61.4 \\
\hline
\end{tabular}

connects the collector and the WT test section and also serves as a channel of bearing pressure supply for gage calibration. Electrical leads of the gages are unsoldered for the connector RS32 TV. The gages work together with the 8 ANCh-23 amplifying apparatus on the carrier frequency. The magnetic biasing frequency for the probes is $20 \mathrm{kHz}$.

The gage at $S=0$ purposed for the measurement of the pressure in the stagnation point of the model has special thermal protection - a plug which presents a tangle of copper wire of $0.08 \mathrm{~mm}$ located in the channel of the receiving duct of the gage.

\section{RESULTS OF NUMERICAL INVESTIGATIONS OF THE FLOW AROUND THE MODEL}

The numerical simulation of the stationary laminar flow of the air plasma in the heater for experimental conditions in the WT VAT-104 (TsAGI) was performed on the base of full Navier-Stokes equations and simplified Maxwell equations for the time-averaged amplitude of the tangential component of the HF electric field intensity $\vec{E}(t, x, r)=\vec{E}_{\theta}(x, r) e^{-i \omega t}$ written in the cylindrical system of coordinates with due regard for the axial symmetry of the problem. Here, $\omega=2 \pi f$ is the circular frequency. Plasma radiation was ignored. The electromagnetic field 
is a superposition of the induction coil field and annular plasma currents; it was treated as a monochromatic one, with the assigned frequency $f$ which depends on the HF current in the inductor. For the calculation, the real inductor was replaced by four infinitely thin ring turns.

The source terms (the Lorentz force and Joule heat release) involved in the Navier-Stokes equations were expressed via the tangential component of the complex amplitude of the curl electric field.

The calculation of the HF electromagnetic field was conducted together with the calculation of the gasdynamic equations on the base of the local one approximation of the Maxwell equations. The followings assumptions were used:

- the plasma is quasi-neutral;

- the magnetic permeability of the plasma is $\mu=1$;

- the dielectric permeability of the plasma does not depend on the electromagnetic field and, hence, does not depend on the coordinates;

- the bias current can be ignored;

- the variation of the electric field in the axial direction can be ignored as compared to its variation in the radial direction:

$$
\frac{\partial}{\partial x} E_{\theta} \ll \frac{\partial}{\partial r} E_{\theta}
$$

The latter assumption leads to the locally one-dimensional approximation for the Maxwell equations, and it significantly simplifies the task. The corresponding equation for determining the tangential component of the curl electric field and boundary condition for this equation are given in $[7,8]$ where the flow of the equilibrium air plasma was considered.

During the combined calculation of the flow and electromagnetic field in the heater of VAT-104 WT (TsAGI), the power $N_{\mathrm{pl}}$ applied to the discharge was assigned as a governing parameter; the respective current in the inductor was determined during the solution process. In turn, the value of $N_{\mathrm{pl}}$ was found with the aid of the generator power $N_{\text {ap }}$ measured experimentally, by the anode supply multiplied by the plasmatorch efficiency.

Figure 8 presents the calculated distributions of the static temperature and Mach number; the data illustrate the pattern of the flow around the blunt cone model at the static pressure of the flow in the output cross section of the nozzle; the pressure is somehow above the pressure in the WT test section.

The pressure ratio on the cone surface $P_{w}$ to the total pressure $P_{0}$ (in the heater) at the nose part of the model (at $S \leq 80 \mathrm{~mm}$, inside the "Mach cone" of the underexpanded jet) is independent of the working chamber pressure $P_{\mathrm{wc}}$ (Fig. 9). At the same time, the ratio of the pressure on the model surface $P_{w}$ to 
Static temperature, $T / \mathrm{K}$
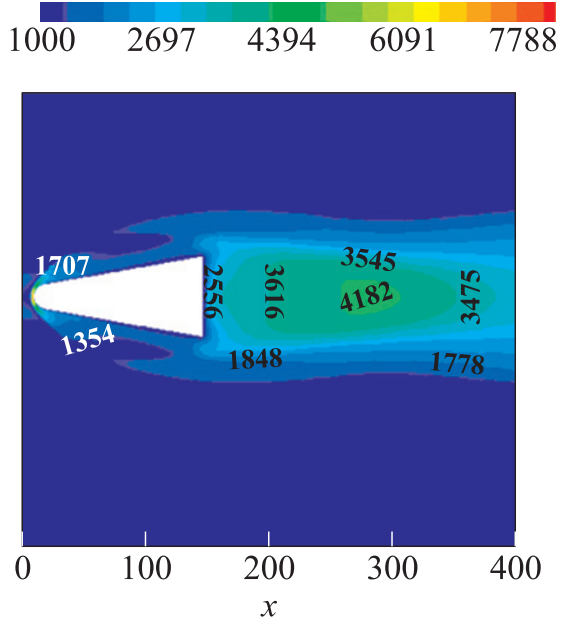

Mach number, $\mathrm{M}$
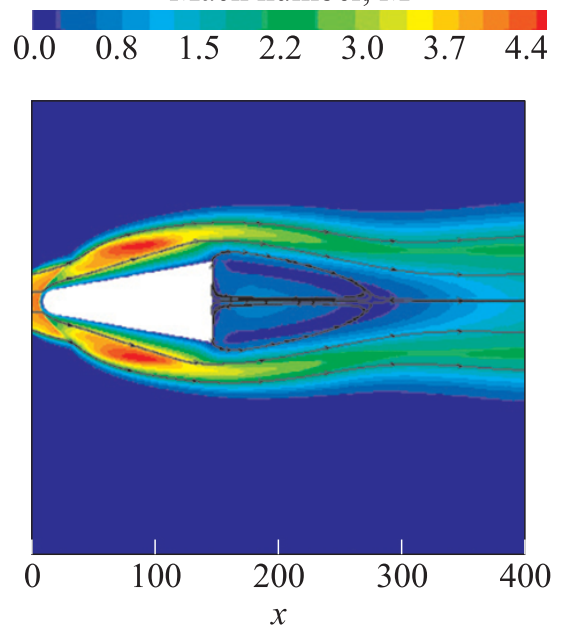

Figure 8 Flow structure near the blunt cone immersed in a supersonic flow

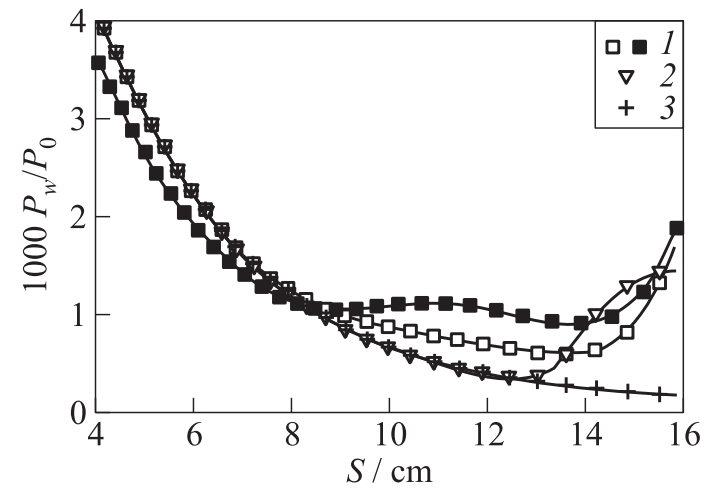

Figure 9 Dependences of the ratio of the pressure at the cone surface to the total pressure $P_{w} / P_{0}$ for various pressure values in the working chamber $(G=3 \mathrm{~g} / \mathrm{s}$ and $\left.W_{a}=205 \mathrm{~kW}\right): 1-P_{\mathrm{wc}}=130 \mathrm{~Pa} ; 2-65$; and $3-P_{\mathrm{wc}}=10 \mathrm{~Pa}$. Filled signs refer to $X_{s}=20 \mathrm{~mm}$ and all other signs refer to $X_{s}=10 \mathrm{~mm}$

the total pressure $P_{0}$ decreases with increasing distance from the nozzle section exit to the stagnation point of the model $X_{s}=L$ (see Figs. 9 and 10).

Specifically the pressure ratio in the stagnation point $S=0$ (stagnation pressure) $P_{s}$ to the total pressure $P_{0}$ is changed sufficiently. 


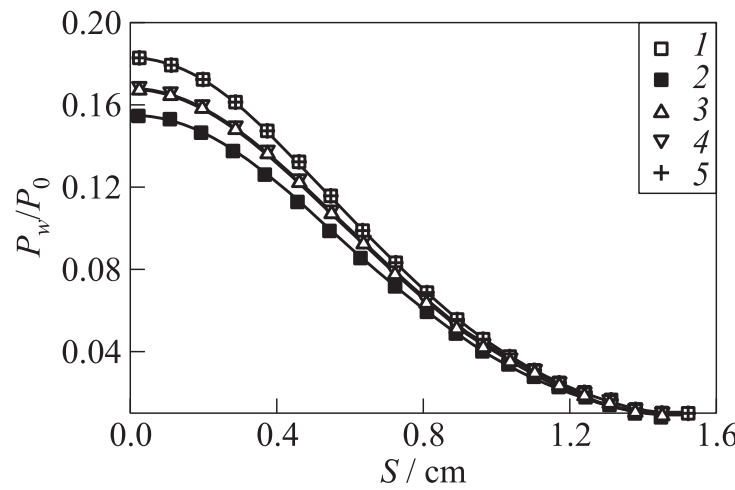

Figure 10 Dependences of the ratio of the pressure at the nose part of the model to the total pressure $P_{w} / P_{0}$ for various parameters: $1-G=3 \mathrm{~g} / \mathrm{s}, W_{a}=205 \mathrm{~kW}, X_{s}$ $=10 \mathrm{~mm}, P_{\mathrm{wc}}=130 \mathrm{~Pa} ; 2-G=3 \mathrm{~g} / \mathrm{s}, W_{a}=205 \mathrm{~kW}, X_{s}=20 \mathrm{~mm}, P_{\mathrm{wc}}=130 \mathrm{~Pa}$; $3-G=2.16 \mathrm{~g} / \mathrm{s}, W_{a}=225 \mathrm{~kW}, X_{s}=15 \mathrm{~mm}, P_{\mathrm{wc}}=130 \mathrm{~Pa} ; 4-G=2.16 \mathrm{~g} / \mathrm{s}$, $W_{a}=200 \mathrm{~kW}, X_{s}=15 \mathrm{~mm}, P_{\mathrm{wc}}=130 \mathrm{~Pa}$; and $5-G=3 \mathrm{~g} / \mathrm{s}, W_{a}=205 \mathrm{~kW}$, $X_{s}=10 \mathrm{~mm}, P_{\mathrm{wc}}=10 \mathrm{~Pa}$

\section{EXPERIMENTAL INVESTIGATION OF THE HEAT FLUX AND PRESSURE DISTRIBUTIONS OVER THE MODEL SURFACE}

The experiment sequence was the following: the model was installed on a holder fastened into a fast-acting mechanism of model introduction in the flow. Before being introduced into the flow, the model axis was parallel to the nozzle axis and $160 \mathrm{~mm}$ far from it, the stagnation point of the model was at the distance of $X=10 \mathrm{~mm}$ from the nozzle exit section.

Then, the WT test section was sealed and pumped out. As the pressure of 1$100 \mathrm{~Pa}$ was reached, the plasmatorch generator was actuated. The discharge was ignited, and smooth mode onset was carried out via increased anode power of the heater $W_{a}$ and air pressure ahead of the throttle (pressure in the settling chamber $\left.P_{\mathrm{fk}}\right)$. The duration of the mode onset was about 50-80 s. As the necessary parameters $\left(W_{a}\right.$ and $\left.P_{\mathrm{fk}}\right)$ were reached, the operator introduced the model in the flow. The heater was switched off in 2-3 s after introducing the model in the flow.

\subsection{Heat Flux Distribution}

As an example, let consider the results of one of the tests (Fig. 11). In this figure, the dependences of the pressure in the settling chamber and the anode 


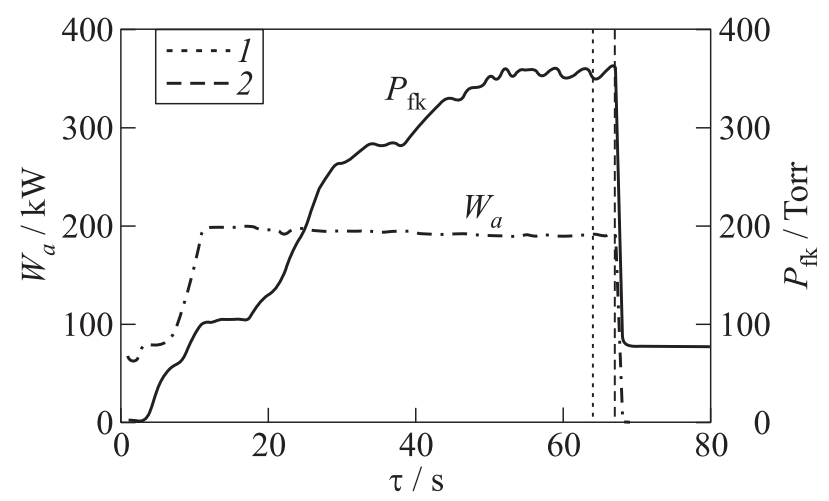

Figure 11 Dependence of the pressure in the settling chamber and the anode power of the heater on time: 1 - input of the model; and 2 - heating off

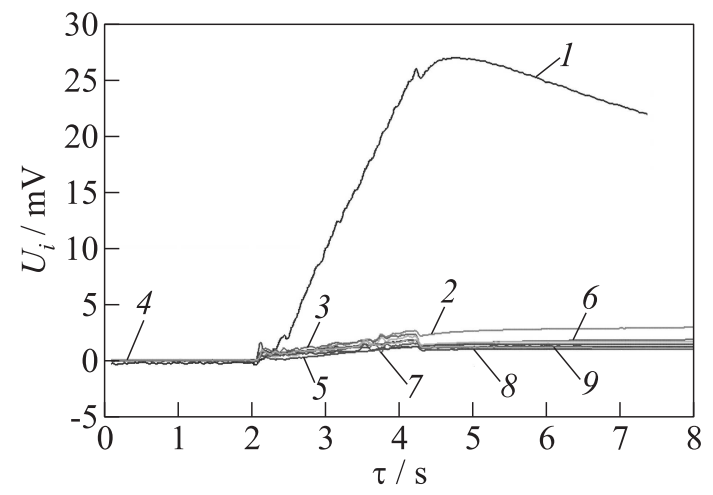

Figure 12 Characteristic records of the thermocouple signals in time for all calorimeters in one of the tests: $1-U_{1} ; 2-U_{2} ; 3-U_{3} ; 4-U_{4} ; 5-U_{5} ; 6-U_{6} ; 7-U_{7}$; $8-U_{8} ;$ and $9-U_{9}$

power of the heater on time are presented. The pressure in the settling chamber was $P_{\mathrm{fk}}=355$ Torr (the pressure in the settling chamber $P_{\mathrm{fk}}$ practically does not differ from the total pressure $P_{0}$ ), the heater power was $W_{a}=190 \mathrm{~kW}$, and the air flow rate was $G=2.5 \mathrm{~g} / \mathrm{s}$.

The characteristic records of the thermocouple signals $U_{i}$ in time for all calorimeters in one of the tests are shown in Fig. 12. Here, the curves numbers correspond to the heat flux probe numbers (see Table 1).

The resulting graph of the heat flux distribution $q_{w}$ over the model length is presented in Fig. 13. The anode power of the heater in each run was $W_{a}$ $=195 \pm 5 \mathrm{~kW}$. 


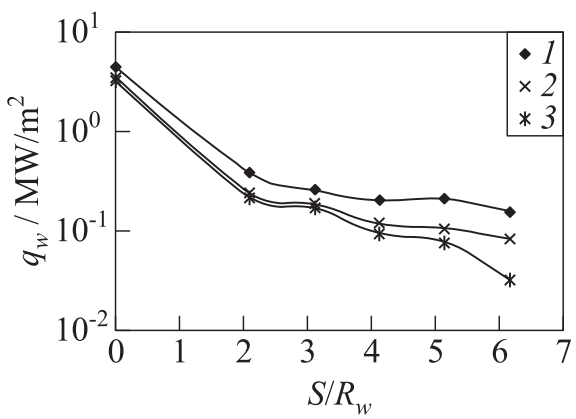

Figure 13 Heat flux distribution over the model length: 1 - 355 Torr; $2-$ 210; and $3-120$ Torr

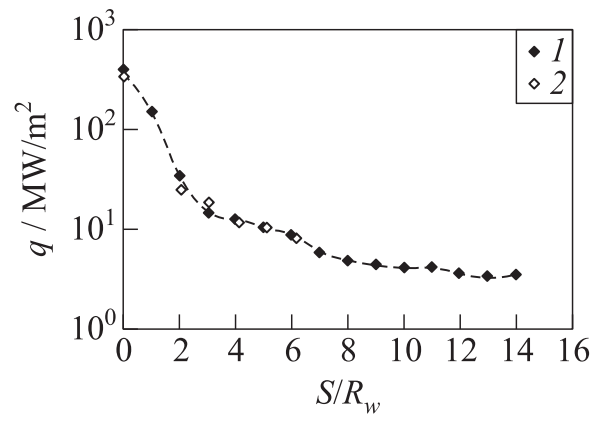

Figure 14 Heat flux distribution over the model surface: 1 - calculations; and 2 - experiment

From Fig. 14, it follows that the experimental findings and numerical data of the heat flux are in agreement (in the calculations, the ratio of the power applied to the gas flow to the anode power of the heater, as well as some other characteristics, were taken from the evaluations of the known values of similar wind tunnels).

At the moment, the investigations are performed to determine the necessary characteristics of the VAT-104 WT.

\subsection{Pressure Distribution}

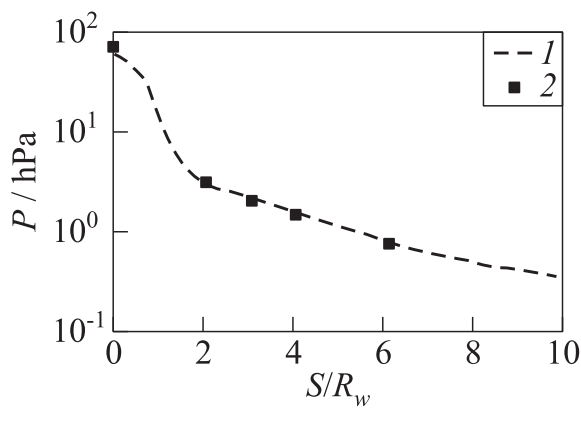

Figure 15 Pressure distribution vs. the distance to the stagnation point: $1-$ calculations; and 2 - experiment
In order to determine the pressure distribution, four tests were carried out at various pressures in the settling chamber and a constant heater power.

Let analyze the results of one test. The settling-chamber pressure was $P_{\mathrm{fk}}=350$ Torr. Figure 15 presents the comparison of the pressure distribution vs. the distance to the stagnation point (experimental value) with the numerical data. It is evident that the pressure obtained in the test is in good agreement with the results of numerical simulation. 


\section{CONCLUDING REMARKS}

The numerical and experimental investigations were carried out to study the flow, heat flux distribution, and pressure distribution over the front and side surfaces of the blunt cone which was in a nonequilibrium high-enthalpy $\left(h_{0}=25 \mathrm{MJ} / \mathrm{kg}\right)$ supersonic $(\mathrm{M}=4)$ air flow. The tests were performed in the VAT-104 WT (TsAGI). The nose part of the model with a small-radius nose $R_{w}=10 \mathrm{~mm}$ and half-angle $\theta=10^{\circ}$ was inside the "Mach cone" of the underexpanded jet flowing out from the WT nozzle. The obtained numerical and experimental data are in qualitative agreement. These results permit to start respective investigations of various methods of the thermal protection of those bodies.

\section{ACKNOWLEDGMENTS}

The authors are deeply indebted to colleagues: N. O. Mjatkovsky, S. M. Pokhvalinsky, A. A. Shamshurin, A. A. Trofimov, and Yu. V. Zhilin for the help in preparation, performance, and analysis of the results of this work.

This work was supported by the Russian Foundation for Basic Research (Grants Nos. 14-08-00971-a and 14-01-00738-a).

\section{REFERENCES}

1. Park, C., J. T. Howe, R. L. Jaffe, and G. V. Candler. 1994. Review of chemicalkinetic problems of future NASA missons. II: Mars entries. J. Thermophys. Heat Transfer 8(1):1994.

2. Zhestkov, B. E., and V. V. Shvedchenko. 1996. Evaluation of material oxidation in induction plasmatron under simulated re-entry conditions. ESA-WPP-103. 204215 .

3. Zhestkov, B. E., D. V. Ivanov, V. V. Shvedchenko, I. V. Jegorov, W. P. P. Fischer, and J. Antonenko. 1999. Calculated and experimental flat and wavy surface temperature distributions. AIAA Paper No. 99-0733.

4. Vasil'evskii, S. A., and A.F. Kolesnikov. 2000. Numerical simulation of equilibrium induction plasma flows in a cylindrical plasmatron channel. J. Fluid Dyn. 35(5):769-777.

5. Afonina, N., V. Cromov, and V. Sakharov. 2004. HIGHTEMP technique for high temperature gas flows simulations. 5th European Symposium on Aerothermodynamics for Space Vehicles ESA SP 563. Noordwijk: ESA Publications Division Cologne ESTEC. 119-123.

6. Sakharov, V., V. Gromov. 2004. CFD modeling of thermally and chemically nonequilibrium flows in discharge channel and in under-expanded plasmatron 
jets over a butt-end probe. 5th European Symposium on Aerothermodynamics for Space Vehicles ESA SP 563. Noordwijk: ESA Publications Division Cologne ESTEC. 323-328.

7. Sakharov, V. I. 2007. Numerical simulation of thermally and chemically nonequilibrium flows and heat transfer in underexpanded induction plasmatron jets. J. Fluid Dyn. 42(6):1007-1016.

8. Sakharov, V.I. 2007. Separation of chemical elements in the discharge channel of an inductive plasma gun. J. Fluid Dyn. 45(4):647-655.

9. Zhestkov, B.E., and V.S. Terent'eva. 2010. Multifunctional coating MAI D5 intended for the protection of refractory materials. Russ. J. Metallurgy 1:33-40.

10. Borovoy, V.Ya., I. V. Egorov, A. S. Skuratov, L. Ya. Paderin, V. V. Shtapov, A. V. Vaganov, E. B. Vasilevskiy, and B. E. Zhestkov. 2011. Experimental researches in high temperature facilities of TsAGI. 4th European Conference for Aerospace Sciences. St. Petersburg. CD. 1184-1859-1DR. Id 602. 